\title{
Beam patterns of terahertz quantum cascade lasers with subwavelength cavity dimensions
}

\author{
A. J. L. Adam, I. Kašalynas, ${ }^{\text {a) }}$ J. N. Hovenier, T. O. Klaassen, and J. R. Gao ${ }^{\text {b) }}$ \\ 1, 2628 CJ Delft, The Netherlands \\ E. E. Orlova \\ Institute for Physics of Microstructures, Russian Academy of Sciences, GSP-105, \\ 603950 Nizhny Novgorod, Russia
}

Kavli Institute of NanoScience Delft, Faculty of Applied Sciences, Delft University of Technology, Lorentzweg

B. S. Williams, S. Kumar, and Q. Hu

Research Laboratory of Electronics, Department of Electrical Engineering and Computer Science, Massachusetts Institute of Technology, Cambridge Massachusetts 02139

\section{J. L. Reno}

Sandia National Laboratories, Department 1123, MS 0601, Albuquerque, New Mexico 87185-0601

(Received 3 December 2005; accepted 6 March 2006; published online 12 April 2006)

\begin{abstract}
The need to reach single-mode lasing and minimize at the same time the electrical dissipation of cryogenically operated terahertz quantum cascade lasers may result in small and subwavelength cavity dimensions. To assess the influence of such dimensions on the shape of the laser emission, we have measured the beam pattern of two metal-metal cavity quantum cascade lasers. The patterns show regular angular intensity variations which depend on the length of the laser cavity. The physical origin of these features is discussed in terms of interference of the coherent radiation emitted by end and side facets of the laser bar. (c) 2006 American Institute of Physics.
\end{abstract}

[DOI: $10.1063 / 1.2194889$ ]

The quest for terahertz sources has resulted recently in the development of the terahertz quantum cascade laser (QCL). ${ }^{1}$ At present, continuous-wave (cw) QCLs in the terahertz range have been demonstrated for frequencies as low as 2.0 THz (Ref. 2) and 1.9 THz (Ref. 3) $(\lambda \approx 160 \mu \mathrm{m})$ and for temperatures up to $117 \mathrm{~K}^{4}$ These sources are very promising as local oscillators for heterodyne detection ${ }^{5,6}$ and for general terahertz imaging applications. ${ }^{7}$ The terahertz QCLs which have achieved the highest temperature performance are based on the so-called "metal-metal waveguides" of subwavelength dimensions. ${ }^{8,9}$ Such waveguides minimize lasing threshold current densities due to their strong confinement of the mode to the gain region, their low losses, and their enhanced facet reflectivities. ${ }^{10}$ Furthermore, the strong confinement has allowed the fabrication of structures with small lateral and transverse dimensions which minimizes electrical power dissipation; this is critical for their cryogenic operation and leads to improved $\mathrm{cw}$ performance. It is expected that the emitted beam from a cavity with subwavelength dimensions would be strongly divergent. ${ }^{10}$ Study of the beam profile therefore is important to characterize this type of terahertz source.

The heterostructure design employed for the terahertz QCLs used in this research is based on resonant longitudinaloptical-phonon scattering to selectively depopulate the lower radiation level. ${ }^{11,12}$ The metal-metal waveguide was fabricated using a copper-to-copper thermocompression bonding technique. ${ }^{8}$ We will report here results of beam profile measurements on two laser samples with subwavelength dimen-

\footnotetext{
${ }^{a}$ Permanent address: Semiconductors Physics Institute, A. Gostauto 11, LT01108 Vilnius, Lithuania.

${ }^{b)}$ Also at SRON Netherlands Institute for Space Research, Utrecht, The Netherlands; electronic mail: j.r.gao@tnw.tudelft.nl
}

sions, fabricated from the same wafer. The metal-metal cavities are bonded to an $n^{+}$GaAs substrate. The front and back facets of the cavities are uncoated. The cavity dimensions and the free space wavelengths are given in Table I, together with the relation between geometry and the Cartesian coordinate system, used to present the experimental data.

Figure 1 shows our experimental setup used to measure the beam patterns. The $n^{+}$GaAs substrate is indium soldered to a copper sample holder, which in turn is attached to the copper cold plate of a helium flow cryostat. The laser bar can be mounted in various orientations with respect to the $50 \mathrm{~mm}$ diameter window at a minimum distance of about $10 \mathrm{~mm}$. It has been carefully checked that the dominant features of the measured beam patterns reported here are not due to spurious reflections inside the cryostat (terahertz absorbers are placed inside) nor to absorption or interference caused by the optical window made out of high density polythene, TPX(4-methyl penten-1) or z-cut quartz. The QCLs are operated in the long-pulsed mode with a $250 \mathrm{~Hz}$ repetition rate and a $1 \mathrm{~ms}$ pulse duration, in order to enable the use of a room temperature pyroelectric detector, together with a lock-in amplifier for noise reduction. The detector, with either a 2 or a $5 \mathrm{~mm}$ diameter sensor, is placed in a two-axis rotation system, with the sensor surface always facing the

TABLE I. Dimensions of the two investigated laser bars and their relation with the coordinate system used, where $W(y)$ is the width, $L(x)$ the length, and $H(z)$ the thickness. Their free space emission wavelengths $\lambda$ are also given.

\begin{tabular}{lcccc}
\hline \hline & $W(y)(\mu \mathrm{m})$ & $L(x)(\mu \mathrm{m})$ & $H(z)(\mu \mathrm{m})$ & $\lambda(\mu \mathrm{m})$ \\
\hline Laser 1 & 25 & 670 & 10 & 102.7 \\
Laser 2 & 25 & 1500 & 10 & 109.1 \\
\hline \hline
\end{tabular}




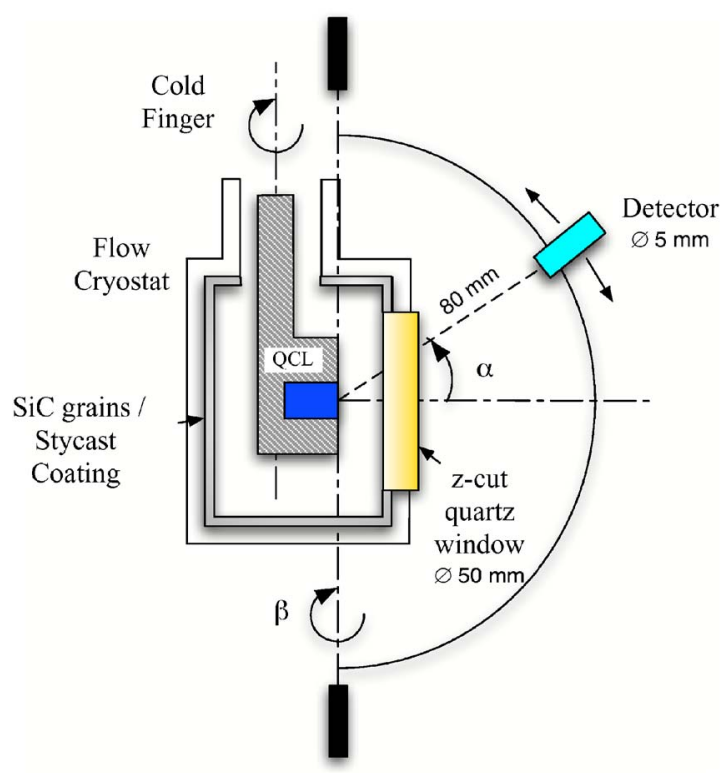

FIG. 1. (Color online) Schematic diagram of the experimental setup for measuring the beam patterns of terahertz quantum cascade lasers.

QCL. The emission has been monitored with the detector at $80 \mathrm{~mm}$ distance from the QCL. The experimental data in Figs. 2 and 3 are presented using the equirectangular projection method.

Figure 2(a) gives the definition of the angular coordinates for the orientation of the samples in the "forward" direction. Figures 2(b) and 2(c) show the measured beam patterns which are characterized by ringlike intensity maxima, centered on the QCL pointing direction $\left(\alpha=\beta=0^{\circ}\right)$. However, the rings are incomplete, appearing only in the upper part of the pattern $\left(\beta>0^{\circ}\right)$. The rings are slightly distorted as a result of the employed equirectangular projection. Between the two samples, the rings in Fig. 2(c) are better distinguished. The angular distance between the first two maximum intensity rings for sample $1\left(\approx 20^{\circ}\right)$ is larger than that for sample $2\left(\approx 9^{\circ}\right)$. It is interesting to notice that the beam patterns do not show a clear intensity maximum in the pointing direction of the laser $\left(\alpha=\beta=0^{\circ}\right)$ as expected.

For the orientation shown in Fig. 3(a), with the top electrode of the QCL facing the window, a set of approximately parallel lines of maximum intensity is observed. Just as for the other orientation, the angular distance between the lines is larger for sample $1\left(\approx 9^{\circ}\right)$ than that for sample $2\left(\approx 5^{\circ}\right)$.

From the results presented above we can conclude that the far field beam pattern of the metal-metal waveguide lasers shows strong intensity modulations where the directions of the extremes form cones around the longitudinal axis of the waveguide. Such patterns are not at all expected from existing information. For example, the terahertz emission was modeled from the front facet of a $10 \mu \mathrm{m}$ thick slab of GaAs of infinite width and length, sandwiched between two metal plates, i.e., a dielectric loaded parallel plate waveguide [situation sketched in Fig. 2(a), but now for infinite dimensions in the $x-y$ plane]. While the radiation from such a guide is highly divergent and, in fact, propagates over a full $2 \pi$ from the aperture, the results show that the output intensity for $\alpha=0$ changes only very slowly and monotonically with the angle $\beta{ }^{8}$ It appears that the experimental data can be understood by modeling such a laser with subwavelength Down (a)

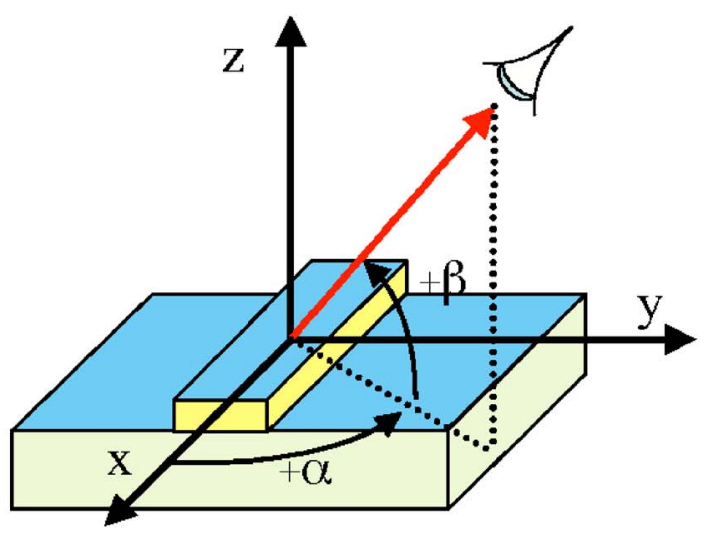

(b)
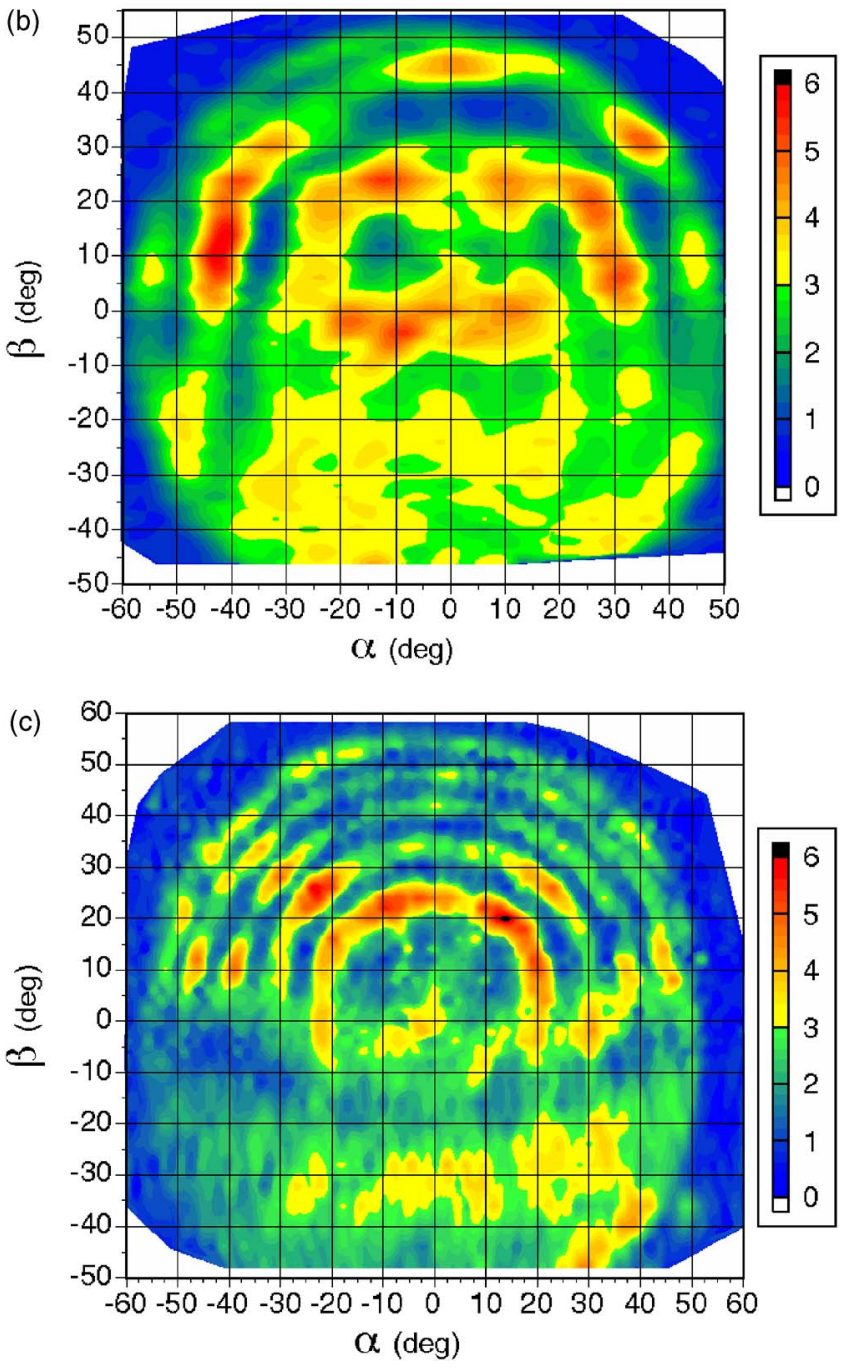

FIG. 2. (Color online) (a) Orientation of samples and definition of angles. [(b) and (c)] Experimental beam patterns of samples 1 and 2 in the "forward" direction. The $x$ axis is perpendicular to the cryostat window.

dimensions as a dielectric antenna. The intensity variations are due to interference of the radiation emitted from different parts, end and side facets of the laser bar. Description of the details of this model, however, is outside the scope of this letter and will be presented elsewhere. ${ }^{13}$ To obtain some insight into the physical origin of these interference patterns, we use an extremely simplified version of this model. We describe the emission of the laser bar with length $L$ as resulting from a system of two coherent point sources situated at the two laser end facets, radiating into free space at a wavelicense or copyright; see http://apl.aip.org/about/rights_and_permissions 
(a)
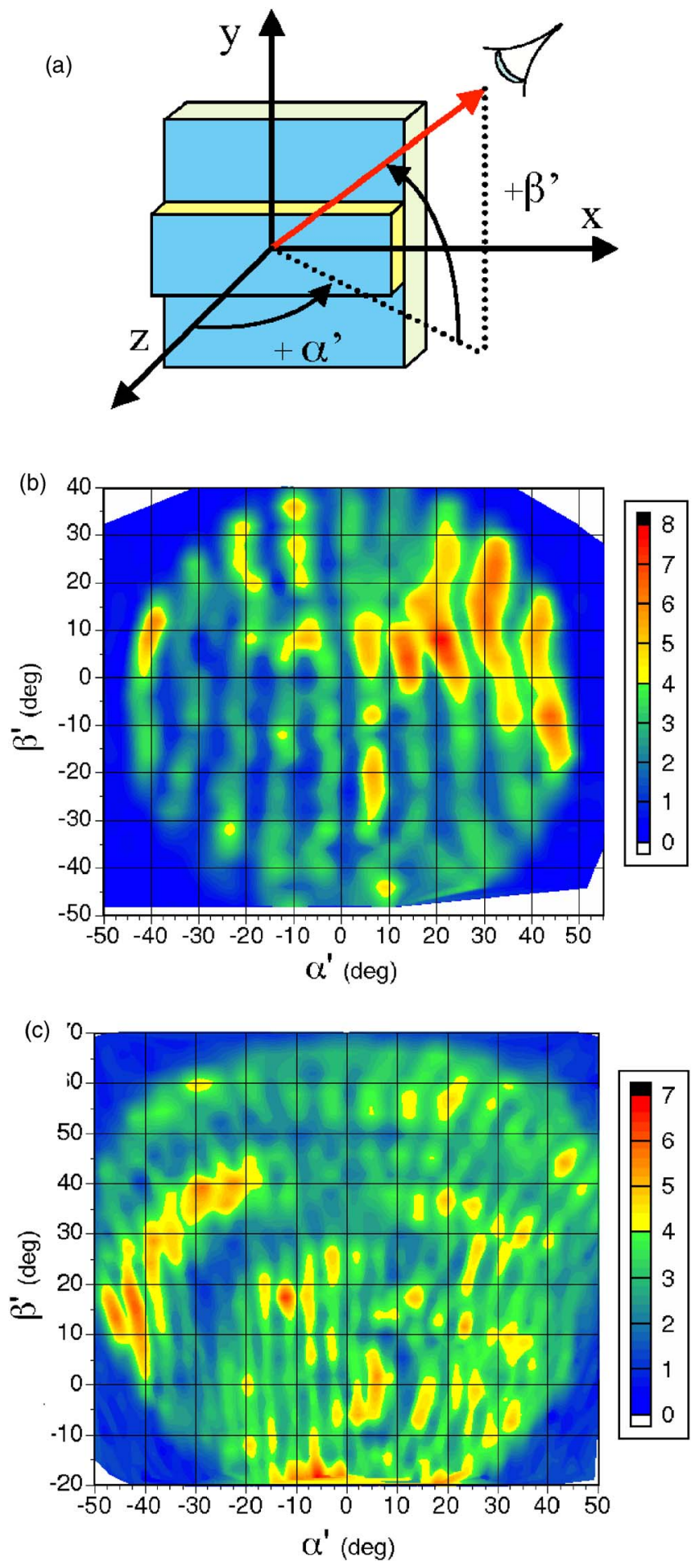

FIG. 3. (Color online) (a) Orientation of samples and definition of new angles $\alpha^{\prime}$ and $\beta^{\prime}$. [(b) and (c)] Experimental beam patterns of samples 1 and 2 in the direction perpendicular to the top electrode; now the $z$ axis $\left(\alpha^{\prime}\right.$ $\left.=\beta^{\prime}=0\right)$ points in the direction of the window.

length $\lambda$. It is readily shown that then in the far field, cone like intensity maxima will occur for directions where $\cos \left(\theta_{m}\right)=k(\lambda / L)$, where $k$ is an integer and $\theta_{m}$ is the angle with respect to the $x$ axis. Using the data on $L$ and $\lambda$ in Table I, the angular distances between the first two maximum intensity rings in Fig. 2 for samples 1 and 2 are calculated to be $17^{\circ}$ and $10^{\circ}$, respectively. For the data in Fig. 3 the angular distances between the maximum intensity lines near $\alpha^{\prime}=0$ are $10^{\circ}$ and $4^{\circ}$, respectively. These values, which are similar to those calculated in Ref. 13, agree quite well with Down simila 12 Aug 2010 to 131.180 .130 .114 . Redistribution subject to AlP the experimental data, and account for the essential interference phenomena observed. It can also be understood that for the beam patterns presented in Fig. 2 for $\beta<0$, a relatively low intensity is observed, because in that part of space the radiation from the rear facet is blocked by the sample holder. It is difficult to obtain accurate information regarding the emission intensity in the forward ( $x$ axis) direction, because for small values of $\theta$, the interference pattern depends critically on the precise values of $L, \lambda$, and the effective index of refraction of the dielectric medium. It must be stressed that, although this "two point source" model reproduces the essential experimental features, a complete description should be based on the antenna model that treats the entire laser bar as a distributed source. ${ }^{13}$

In conclusion, we have experimentally investigated the beam pattern of two different terahertz QCLs with subwavelength cavity dimensions. We discovered that the emitted beam is not only strongly divergent but also shows ringlike patterns resulting from the far field interference of the coherent radiation emitted by all facets of the laser bar. Our experimental results showed that for lasers with deep subwavelength apertures, the conventional method to obtain the far field pattern by Fourier transforming the field at the facet is inadequate. One needs to take all the field distribution into account to understand the interference patterns of the far field.

The authors acknowledge T. M. Klapwijk for his stimulating discussions and support. Two of the authors (A. J. L. A. and I. K.) gratefully acknowledge support through the European Union HP-RTN project "Terahertz Electronics: Components and Systems (INTERACTION)" under Contract No. HPRN-CT-2002-00206. The work at MIT is supported by AFOSR, NASA, and the NSF. Sandia is a multiprogram laboratory operated by Sandia Corporation, a Lockheed Martin Company, for the (U.S.) Department of Energy under Contract No. DE-AC04-94AL85000.

${ }^{1}$ R. Köhler, A. Tredicucci, F. Beltram, H. E. Beere, E. H. Linfield, A. G. Davies, D. A. Ritchie, R. C. Iotti, and F. Rossi, Nature (London) 417, 156 (2002).

${ }^{2}$ C. Worrall, J. Alton, M. Houghton, S. Barbieri, H. Beere, D. Ritchie, and C. Sirtori, Opt. Express 14, 171 (2006).

${ }^{3}$ S. Kumar, B. S. Williams, Q. Hu, and J. L. Reno, Appl. Phys. Lett. 88, 121123 (2006).

${ }^{4}$ B. S. Williams, S. Kumar, Q. Hu, and J. L. Reno, Opt. Express 13, 3331 (2005).

${ }^{5}$ J. R. Gao, J. N. Hovenier, Z. Q. Yang, J. J. A. Baselmans, A. Baryshev, M. Hajenius, T. M. Klapwijk, A. J. L. Adam, T. O. Klaassen, B. S. Williams, S. Kumar, Q. Hu, and J. L. Reno, Appl. Phys. Lett. 86, 244104 (2005).

${ }^{6}$ H.-W. Hübers, S. G. Pavlov, A. D. Semenov, R. Köhler, L. Mahler, A. Tredicucci, H. E. Beere, D. A. Ritchie, and E. H. Linfield, Opt. Express 13, 5890 (2005).

${ }^{7}$ D. R. Chamberlin, P. R. Robrish, W. R. Trutna, G. Scalari, M. Giovanni, L., Ajili, and J. Faist, Appl. Opt. 44, 121 (2005).

${ }^{8}$ B. S. Williams, S. Kumar, Q. Hu, and J. L. Reno, Electron. Lett. 40, 431 (2004).

${ }^{9}$ S. Dhillon, J. Alton, S. Barbieri, C. Sirtori, A. de Rossi, M. Calligaro, H. E. Beere, and D. Ritchie, Appl. Phys. Lett. 87, 071107 (2005).

${ }^{10}$ S. Kohen, B. S. Williams, and Q. Hu, J. Appl. Phys. 97, 053106 (2005).

${ }^{11}$ Q. Hu, B. S. Williams, S. Kumar, H. Callebaut, S. Kohen, and J. L. Reno, Semicond. Sci. Technol. 20, S228 (2005).

${ }^{12}$ B. S. Williams, H. Callebaut, S. Kumar, Q. Hu, and J. L. Reno, Appl. Phys. Lett. 82, 1015 (2003).

${ }^{13}$ E. E. Orlova, J. N. Hovenier, T. O. Klaassen, I. Kašalynas, A. J. L. Adam, J. R. Gao, T. M. Klapwijk, B. S. Williams, S. Kumar, Q. Hu, and J. L. Reno, Phys. Rev. Lett. (unpublished). 